EPJ Web of Conferences 47, 04002 (2013)

DOI: $10.1051 /$ epjconf/20134704002

(C) Owned by the authors, published by EDP Sciences, 2013

\title{
The spectroscopic study of M8.5-M9.5 stars and brown dwarfs
}

\author{
M.K. Kuznetsov ${ }^{1, a}$, Y.V. Pavlenko ${ }^{1,3}$ and M.C. Galvez-Ortiz ${ }^{2,3}$ \\ ${ }^{1}$ Main Astronomical Observatory, Academy of Sciences of Ukraine, Golosiiv Woods, \\ Kyiv-127, 03680, Ukraine \\ ${ }^{2}$ Centro de Astrobiologia (CSIC-INTA). Crta, Ajalvil km 4, 28850 Torrejón de Ardoz, Madrid, \\ Spain \\ ${ }^{3}$ Centre for Astrophysics Research, Science and Technology Research Institute, \\ University of Hertfordshire, Hatfield AL10 9AB, UK
}

\begin{abstract}
We present high-resolution spectra analysis of the three late-M dwarfs LP944-20, SIPS J2045-6332 and DENIS-P J0021.0-4244. The stellar spectra were observed with Very Large Telescope/Ultraviolet-Visual Echelle Spectrograph (VLT/UVES) in optical and near-infrared regions. The effective temperatures $T_{\text {eff }}$ and $\log \mathrm{g}$ was defined by comparing observed and theoretical energy distributions for the investigated objects. Synthetic spectra were calculated for PHOENIX atmosphere models - COND and DUSTY, as well as for Semi-empirical atmosphere model. We discuss the influence of the effects associated with dust in stellar atmosphere on the energy distribution in the stellar spectra.
\end{abstract}

\section{INTRODUCTION}

Brown dwarfs are objects occupying an intermediate position between stars and giant planets. Investigation of brown dwarfs is important for understanding the evolution and structure of stars and planets. We present a study of 3 late-M dwarfs LP944-20, SIPS J2045-6332 and DENIS-P J0021.04244 , that could be young brown dwarfs.

LP944-20 is an archetypical young brown dwarf. The spectral type of LP 944-20 is estimated as M9.0 [3]. The object is a member of the Castor group [12]. The spectral type of SIPS J2045-6332 range M8.5-M9.0 [15]. DENIS-P J0021.0-4244 is a member of wide ultracool binary with the spectral type M9.5 [6]. The mass is estimated as $M=0.079 \pm 0.004 M_{\odot}$ [4]. According to previous study the metallicity of the objects is close to Solar metallicity (see [7]).

Our sample was observed with the UVES spectrograph on the Very Large Telescope (VLT). The spectra were obtaned with resolution $\mathrm{R}=38000$ in the spectral regions 5700-7530 $\AA$ and 7650-9470 $\mathrm{A}$. The spectra were extracted using the standard reduction procedures in $\mathrm{IRAF}^{1}$.

The comparison of high-resolution spectroscopy with synthetic models of the atmospheres of stars provides a powerful tool to study their properties (chemical composition, effective temperature, surface gravity and rotational velocity).

This work is part of an effort to characterize a sample of nearby M-dwarfs, from a program of Moving Groups research. A detailed study of the Moving Groups (MG) is reported in these proceedings (Galvez-Ortiz et al.).

\footnotetext{
ae-mail: maxk.kuznetsov@gmail.com

${ }^{1}$ IRAF is distributed by the National Optical Observatory, which is operated by the Association of Universities for Research in Astronomy, Inc., under contract with the National Science Foundation.

This is an Open Access article distributed under the terms of the Creative Commons Attribution License 2.0, which permits unrestricted use, distribution, and reproduction in any medium, provided the original work is properly cited.
} 


\section{STANDART SPECTRAL MODELLING AND SYNTHETIC SPECTRA}

We first performed a comparison with Synthetic spectra that were calculated by the program WITA6 [10] from the DUSTY and COND model atmospheres [1] of solar [2] metallicity. Synthetic spectra were computed for models with effective temperatures $T_{\text {eff }}=2100-2500 \mathrm{~K}$ with a step of $100 \mathrm{~K}$ and $\log g=4.0-5.5 \mathrm{~cm} * s^{-2}$ with a step of $0.5 \mathrm{~cm} * s^{-2}$. The calculation was assuming local thermodynamic equilibrium (LTE) for a one-dimensional atmosphere without sources of energy in hydrostatic equilibrium. In the work we used the VALD atomic lines list [13] and the TiO lines list from [11]. The rotational velocity of the objects were taken from [7]. Unfurtunately, none of parameters set allowed us to achieve a good fitting for the given spectral range.

\section{SEMI-EMPIRICAL MODEL ATMOSPHERE}

Since the presence of dust plays important role in the formation of late M dwarfs spectra (see [17], [8]), we tried a different approach to find the best fit for targets. TiO and $\mathrm{VO}$ absorption bands are very sensitive to dusty effects such as decrease of the molecules abundance in gas condition due to the molecules condensation on the dust particles and radiation scattering in the dust clouds. We used semi-empirical model of atmospheres based on DUSTY and COND models (see [9]) to include the additional effects of dust formation. Semi-empirical model based on DUSTY and COND models were developed. We assume that dust clouds are located in the uppermost layers of stellar atmosphere and do not affect the distribution of temperatures and pressures. The dust opacity originates in the shell-like structures lying above the photosphere [9]. Decreasing of $\mathrm{TiO}$ and $\mathrm{VO}$ molecules concentration and radiation scattering in the dust clouds was modelled by four parameters: the number of the layer in the model, above which there is no absorption of $\mathrm{TiO}$ and $\mathrm{VO}$, coefficient of molecular concentration reduction, the optical thickness of the dust cloud and the location of the maximum of opacity of the clouds in the atmosphere. See [9] and [14] for a more detailed description of the method. Both for DUSTY and for COND models were modified. The grid of synthetic spectra based on semi-empirical models (for DUSTY and COND) was calculated for effective temperatures $T_{\text {eff }}=2100-2500 \mathrm{~K}$, $\log g=4.0-5.5 \mathrm{~cm} * \mathrm{~s}^{-2}$ and for different sets of free parameters associated with dust.

\section{FIT TO THE OBSERVED SPECTRAL ENERGY DISTRIBUTION}

We obtained fundamental parameters of LP944-20, SIPS J2045-6332 and DENIS-P J0021.0-4244 based on comparison between observed and synthetic spectral energy distribution in the spectral regions 5700-7530 $\AA$ and 7650-9470 $\mathrm{A}$. The TiO and VO absorption bands are dominant opacity source in this regions. They are sensitive to effective temperature changing but they feel little change of $\log g$. The subordinate doublet profile of $\mathrm{Na} \mathrm{I} \sim 8190 \AA$ isn't sensitive for $T_{\text {eff }}$ and $\log g$ in case of late-M dwarfs. While the resonance doublet $\mathrm{K} \mathrm{I} \sim 7700 \AA$ is sensitive to effective temperature and $\log g$. This makes it an effective tool for the study of the stellar fundamental parameters.

The fit for DUSTY, COND and semi-empirical model based on DUSTY and COND were computed independently. The S function analysis described in [9] was used to obtain best fit for each model grid. The minimum value of S-function corresponds to the best fit.

\section{RESULTS}

We carried out our fits for the DUSTY and COND model atmospheres as well as for semi-empirical model based on DUSTY and COND models. The effective temperatures $T_{\text {eff }}$ and $\log \mathrm{g}$ were obtained. The fundamental parameters based on fitting are shown at the Table 1. The semi-empirical model based on DUSTY provides the best fitting for all our objects. 


\section{Hot Planets and Cool Stars}

Table 1. The objects parameters from the high resolution spectra.

\begin{tabular}{|c|c|c|c|c|c|c|c|c|c|}
\hline & \multicolumn{2}{|r|}{$\mathrm{Sp}$} & \multicolumn{3}{|c|}{ DUSTY } & \multicolumn{3}{|c|}{ COND } & \\
\hline Object & $\mathrm{Sp}$ & $T_{e f f}(\mathrm{Sp})$ & $T_{\text {eff }}$ & $\log g$ & {$[\mathrm{M} / \mathrm{H}]$} & $T_{e f f}$ & $\log g$ & {$[\mathrm{M} / \mathrm{H}]$} & best fit \\
\hline lp944-20 & M9.0 & $2300-2500$ & 2400 & 4.0 & 0.0 & 2500 & 4.0 & 0.0 & DUSTY \\
\hline denis 0021 & M9.5 & $2300-$ & 2400 & 4.0 & 0.0 & 2500 & 4.0 & 0.0 & DUSTY \\
\hline sips2045 & M8.5 & $2300-2500$ & 2400 & 4.0 & 0.0 & 2500 & 4.0 & 0.0 & DUSTY \\
\hline
\end{tabular}
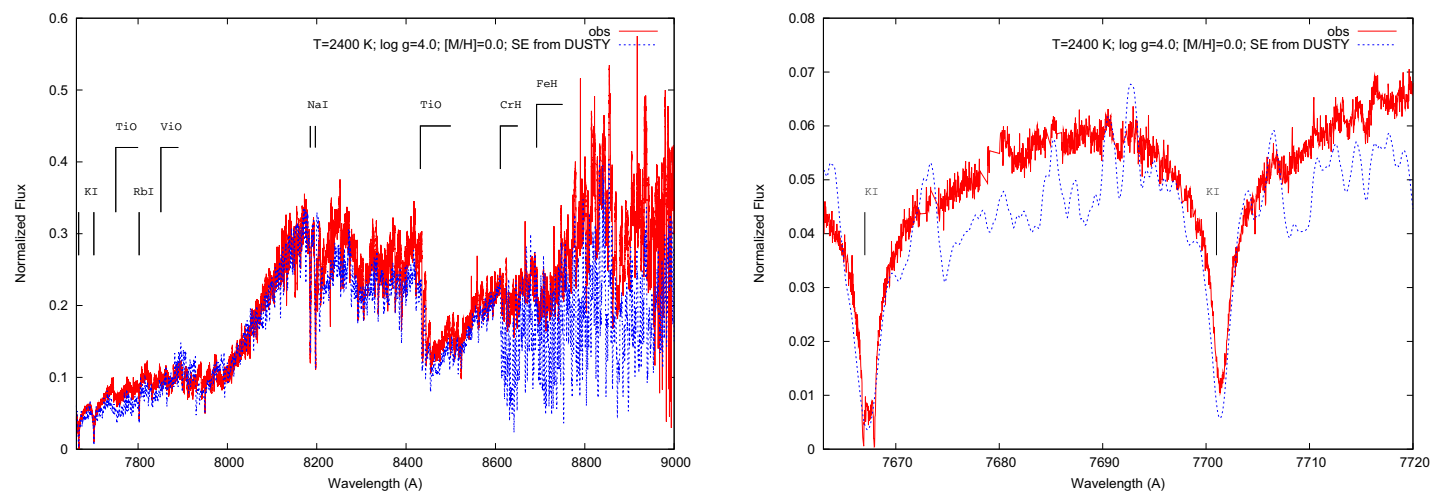

Figure 1. Left: the best fit of LP944-20 for spectral region across $7650-9000 \AA$ A. Right: the region of KI 7700 dublet.
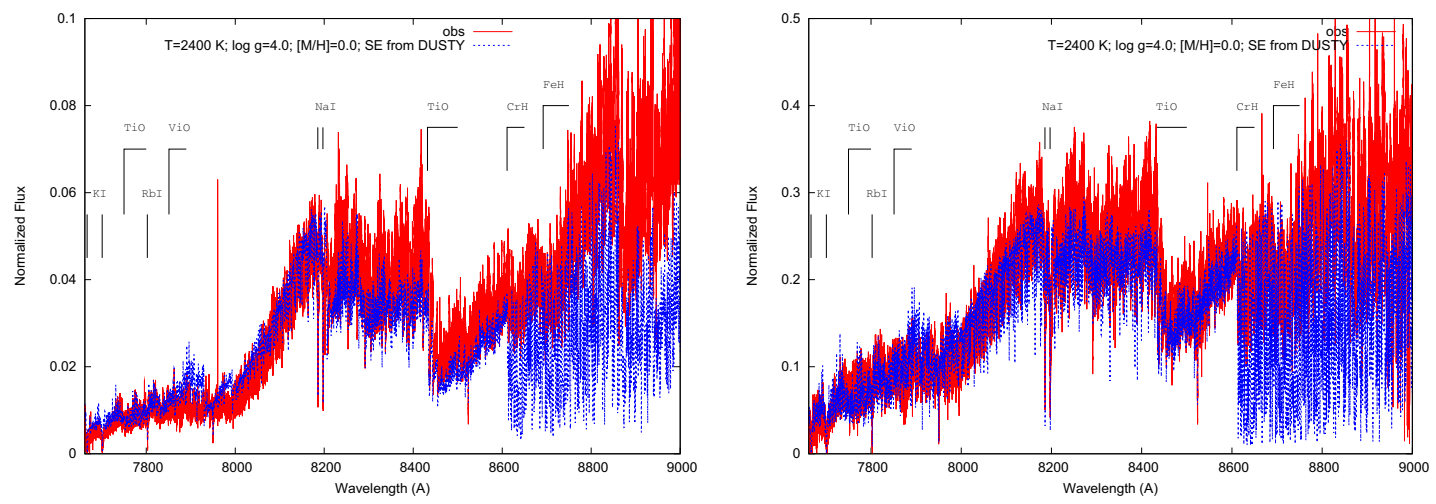

Figure 2. The best fitting for SIPS J2045-6332 (left) and DENIS-P J0021.0-4244 (right) in spectral region across 7650-9000 А.

The spectral classes in the Table 1 comes from [7]. It's difficult to associate effective temperature and spectral class for stars of spectral type M8.5-M9.5 (see [16]). In the Table 1 we show the range of effective temperatures from [5]).

The Figs. 1 and 2 shows comparison between observed and synthetic spectra across the region 7650-9000 A. The use of semi-empirical model allows us to obtain good conformity between theoretical and observed spectra. There are significant difficulties with modeling region across $8600-9000 \AA$. We believe that this can be consistent with the difficulty in flux calibration and telluric lines correction. 
EPJ Web of Conferences

M.K. Kuznetsov and Ya.,V. Pavlenko work was supported by EU PF7 Marie Curie Initial Training Networks (ITN) ROPACS project (GA N 213646).

\section{References}

[1] Allard F., Hauschildt P.H., Alexander D.R., Tamanai A., Schweitzer A., ApJ, 556, 357 (2001)

[2] Anders E., Grevesse N., Geochim. Cosmochim. Acta 53, 3273 (1989)

[3] Basri G., Mohanty S., Allard F., Hauschildt P. H., Delfosse X., et al., ApJ, 538, 363 (2000)

[4] Caballero, J.A., A\& A, 462, 61 (2007)

[5] Dahn, C.C., Harris, H.C., Vrba, F.J., Guetter, H.H., et al., AJ, 124, 1170 (2002)

[6] Delfosse X., Tinney C.G., Forveille T., Epchtein N., A\& AS, 135, 41 (1999)

[7] Gálvez-Ortiz M.C., Clarke J.R.A., Pinfield D.J., Jenkins J.S., MNRAS, 409, 552 (2010)

[8] Hauschildt P. Allard F., ApJ, 512, 377 (1992)

[9] Pavlenko Ya.V., Jones H.R.A., Martín E.L., Guenther E., MNRAS, 380, 3, 1285 (2007)

[10] Pavlenko Ya. V., Ap \& SS, 253, 43 (2007)

[11] Plez B. A\& A, 337, 495 (1998)

[12] Ribas I. A\& A, 400, 297 (2003)

[13] Kupka F., Piskunov N., Ryabchikova T.A. et al., A\& AS, 138, 119 (1999)

[14] Kuznetsov M.K., Pavlenko Ya. V., Galvez-Ortiz M.C., KFNT, 28, 6, 32 (2012)

[15] Reid I.N., Cruz K.L., Kirkpatrick J.D., Allen P.R., et al., AJ, 136, 1290 (2007)

[16] Reylé C., Rajpurohit A.S., Schultheis M., Allard F., ASPC, 448, 929 (2011)

[17] Tsuji T., Ohnaka K., Aoki W., A\& AS, 305, L1 (1996) 